\title{
Diseño de reingeniería del sistema de puesta a tierra de un transformador de 300 KVA
}

\section{Re-engineering desing of the grounding system of a $300 \mathrm{KVA}$ transformer}

DOMINGUEZ-DE LA CRUZ, Joel†', MARTÍNEZ-RODRÍGUEZ, Guillermo Miguel, GARRIDOMELENDEZ, Javier y JIMÉNEZ-RIVERA Jesús

Universidad Veracruzana, Facultad de Ingeniería

ID $1^{\text {er }}$ Autor: Joel, Dominguez-De La Cruz / ORC ID: 0000-0003-3574-3480, arXiv Author ID: joeldguez89, CVU CONACYT ID: 1001051

ID $1^{\text {er }}$ Coautor: Guillermo Miguel, Martínez-Rodríguez / ORC ID: 0000-0001-9760-7893, arXiv Author ID: Guillermo\#2, CVU CONACYT ID: 947594

ID $2^{\text {do }}$ Coautor: Javier, Garrido-Melendez / ORC ID: 0000-0001-9143-408X, Researcher ID Thomson: C-9373-2018

ID $3^{\text {er }}$ Coautor: Jesús, Jiménez-Rivera / ORC ID: 0000-0002-3134-6405, Researcher ID Thomson: T-2722-2018, CVU CONACYT ID: 599539

DOI: $10.35429 / J T E N .2019 .9 .3 .1 .7$

Recibido 03 de Enero, 2019; Aceptado 30 Marzo, 2019

\section{Resumen}

Se presenta un diseño de reingeniería de un sistema de puesta a tierra (SPT) de un transformador de 300 KVA de la Universidad Veracruzana, como antecedente se hicieron mediciones al transformador con un analizador de calidad de energía, de los datos medidos y analizados se detectaron altos valores de armónicos en el neutro, se deduce que el problema es en el SPT, para verificar lo anterior se realizaron mediciones con un equipo probador de electrodos de conexión a tierra, obteniendo alta resistencia en las varillas del SPT, la cual no cumple con la NOM-001SEDE-2012, además se simularon los potenciales de contacto y paso con ayuda del software ETAP mostrando una diferencia de potencial peligrosa para el ser humano, se propone una metodología para el diseño y reingeniería del SPT del transformador mediante análisis del sistema actual, cálculos y simulaciones utilizando el software ETAP, obteniendo valores que cumplan con la normativa y lo más importante que brinden seguridad a las personas y equipos.

\section{Puesta a tierra, Armónicos, Neutro}

\begin{abstract}
Resumen
Re-engineering design of grounding system (GS) is presented of a $300 \mathrm{KVA}$ transformer from the Universidad Veracruzana, as a background measurements were made to transformer with an power quality analyzer, from the measured and analyzed data high values were detected of harmonics in the neutral, we deduce that the problem is in the GS, to verify the above, measurements were made with a grounding electrode tester, obtaining high resistance in the rods of GS, this value fail with respect to norm NOM -001-SEDE-2012, also, the contact and step potentials were simulated with the help of ETAP Software showing a dangerous potential difference for the human being, a methodology is proposed for the design and reengineering of SG of the transformer through actual system analysis, calculations and simulations using ETAP software, obtaining values that comply with the normative and most importantly that provide security to people and equipment's .
\end{abstract}

\section{Grounding, Harmonics, Neutral}

Citación: DOMINGUEZ-DE LA CRUZ, Joel, MARTÍNEZ-RODRÍGUEZ, Guillermo Miguel, GARRIDO-MELENDEZ, Javier y JIMÉNEZ-RIVERA Jesús. Diseño de reingeniería del sistema de puesta a tierra de un transformador de 300 KVA. Revista de Ingeniería Tecnológica. 2019. 3-9: 1-7

$\uparrow$ Investigador contribuyendo como primer autor. 


\section{Introducción}

Un sistema de puesta a tierra (SPT) comprende todas las instalaciones de conexión a tierra interconectadas en un área específica [1]. Sin un sistema de puesta a tierra eficaz, podríamos vernos expuestos a riesgos de descargas eléctricas, problemas de distorsión de armónicos y problemas de factor de potencia [3].

La universidad Veracruzana cuenta con un transformador de $300 \mathrm{KVA}$ el cual se analizó la calidad de energía, encontrándose fallas en el neutro. Con los datos obtenidos de las mediciones se deduce que el motivo de la falla es el SPT. Se realiza un diseño de reingeniería para el SPT con el objetivo de mejorar la seguridad en las personas alrededor del equipo y la seguridad de este.

En este trabajo, en la sección dos, se explica el análisis del sistema eléctrico con los datos obtenidos de las mediciones, así como los comportamientos de las ondas sinusoidales del transformador, el diagrama fasorial y armónicos. En la sección tres se analiza el SPT, se simula los potenciales de contacto y de paso mediante el software ETAP. En la última sección se realiza un diseño del SPT con cálculos y simulaciones con el software ETAP.

\section{Análisis del sistema del sistema eléctrico actual}

El transformador para analizar se encuentra ubicado en el lado oriente de la Universidad Veracruzana de $300 \mathrm{kVA}, 13.2 \mathrm{kV} / 220-127 \mathrm{~V}$ con una conexión delta-estrella $\Delta$-Y tipo ON$\mathrm{AN}$; con impedancia de $\mathrm{Z}=4.6 \%$ marca DALTON.

Se realizaron mediciones con el analizador de calidad de energía Fluke 435-II. Con los datos obtenidos se realizan los siguientes análisis:

- $\quad$ Forma de onda de Voltajes y Corrientes.

- Diagrama fasorial

- Armónicos

\section{Análisis de Forma de onda de V y I}

En la Figura 1 se muestra el comportamiento de las ondas sinusoidales de voltaje en las fases del transformador, se puede observar que las ondas no presentan ninguna distorsión.
En la Figura 2 y 3 se muestra la forma de onda sinusoidal de voltaje y corriente del neutro respectivamente, se observa que las ondas presentan distorsión en ambas.

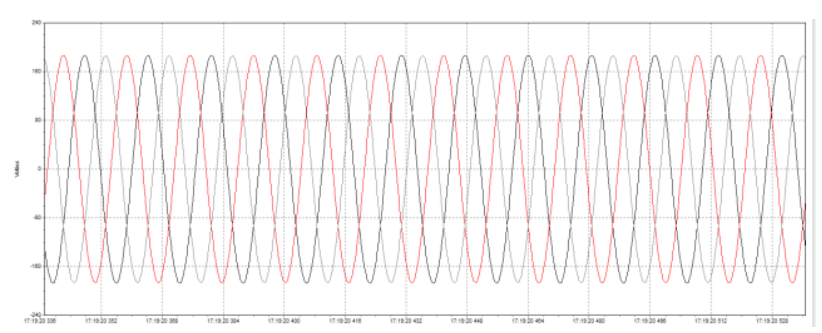

Figura 1 Ondas sinusoidales de voltaje en fases Fuente: Elaboración Propia

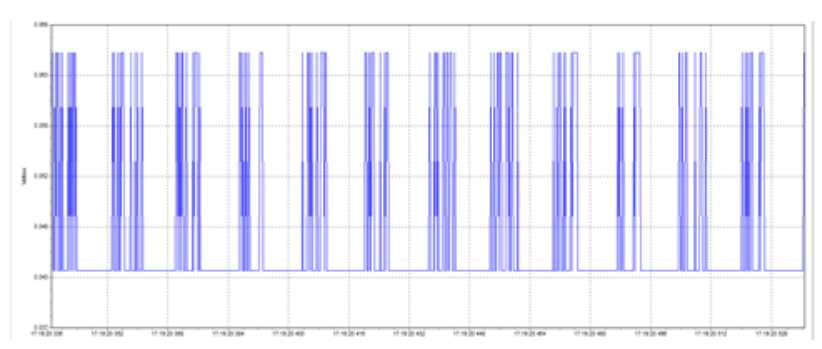

Figura 2 Onda sinusoidal de voltaje en el neutro Fuente: Elaboración Propia

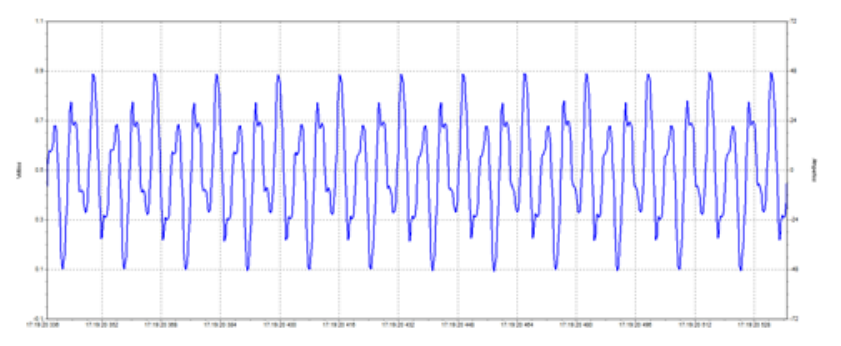

Figura 3 Onda sinusoidal de corriente en el neutro Fuente: Elaboración Propia

\section{Análisis del diagrama fasorial}

En la Figura 4 se muestra el diagrama fasorial de corriente, se puede observar un desbalance de:

- $\quad$ Fase A muestra desbalance de $-7^{\circ}$

- $\quad$ Fase B muestra desbalance de $-7^{\circ}$

- $\quad$ Fase $\mathrm{C}$ muestra desbalance de $-2^{\circ}$

Una de las causas de tener un aumento de corriente en el neutro es por desbalance en las cargas [5]. Con base en los valores de desbalance en las fases se descarta la posibilidad de que la corriente en el neutro sea ocasionada por este. 


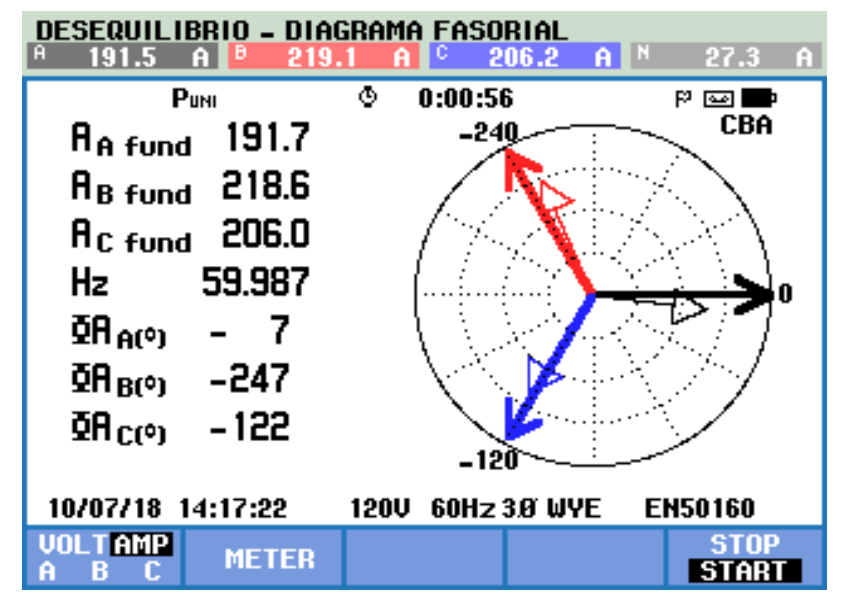

Figura 4 Diagrama de espectros de armónicos de corriente Fuente: Elaboración Propia

\section{Análisis de Armónicos}

En la figura 5 se muestra el diagrama de espectros de armónicos de voltaje de las fases. En las fases se obtuvo un THD del $0.4 \%$ de armónicos de voltaje, en la Figura 6 se muestra el diagrama de espectros de armónicos de voltaje en el neutro, obteniendo un THD de 95.6\%, se puede notar que sobresalen los armónicos de secuencia cero, los cuales afectan al neutro [4].

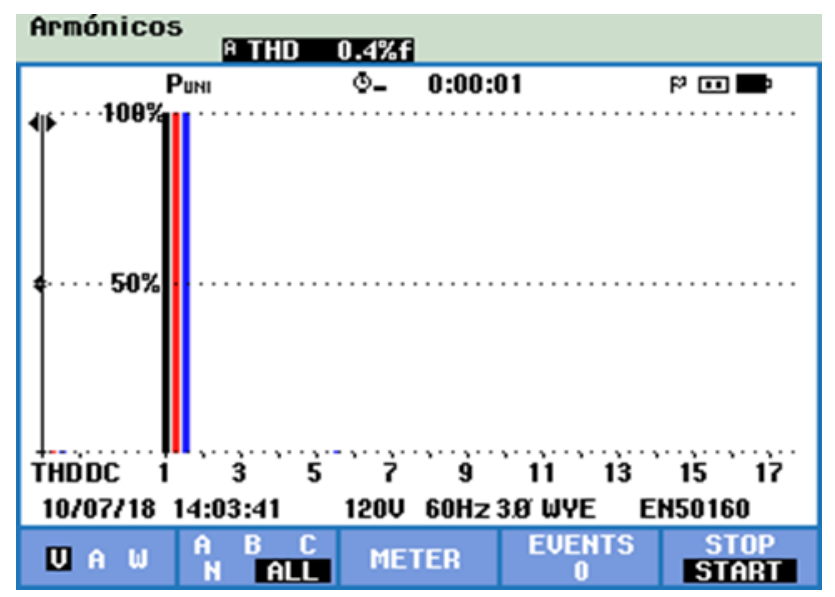

Figura 5 Diagrama de espectros de armónicos de voltaje Fuente: Elaboración Propia

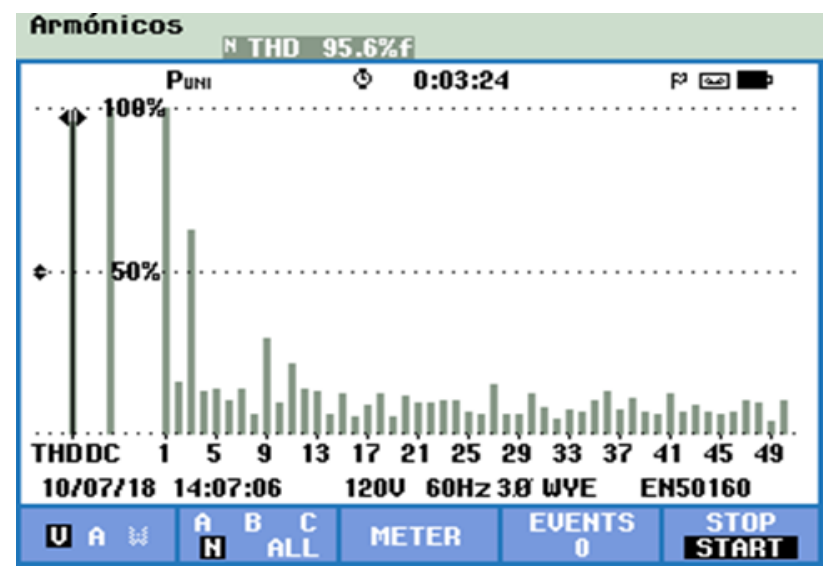

Figura 6 Diagrama de espectros de armónicos de voltaje en el neutro

Fuente: Elaboración Propia

ISSN 2523-6776

ECORFAN® Todos los derechos reservados
En la Figura 7 se muestra el diagrama de espectros de armónicos de corriente en el neutro, la cual muestra un THD de $231.7 \%$, los armónicos más sobresalientes son los de secuencia cero.

Con los datos obtenidos podemos concluir que el aumento de corriente es debido a las cargas no lineales que generan armónicos de secuencia cero. Las cargas no lineales son aquellas donde su impedancia está en constante cambio de acuerdo con la tensión aplicada, esto significa que la corriente consumida por la carga no lineal no será sinusoidal, en otras palabras, la corriente que fluye a través de ellas no se comporta de acuerdo con la ley de $\mathrm{Ohm}$

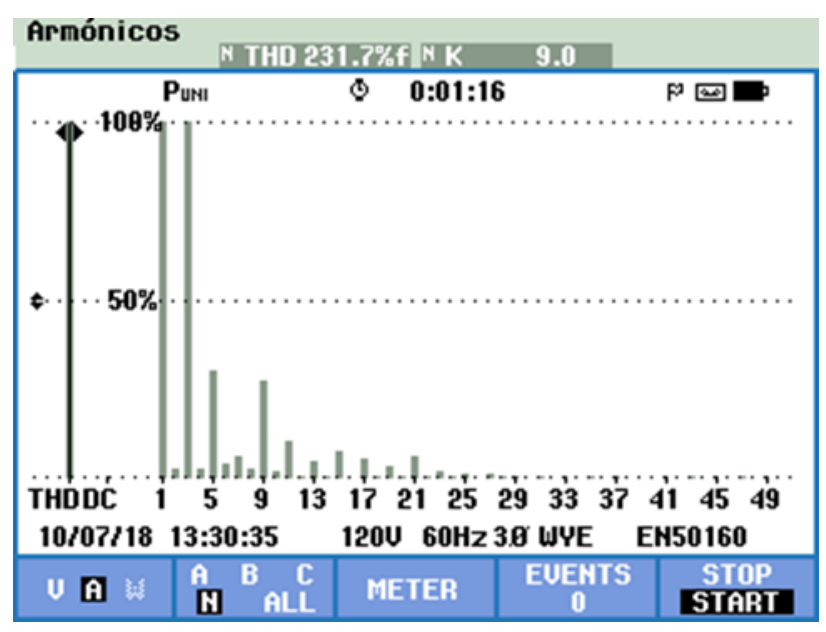

Figura 7 Diagrama de espectros de armónicos de corriente en el neutro

Fuente: Elaboración Propia

Para comprobar que una carga no lineal se pueden inyectar armónicos de secuencia cero (3ro, 9no, 15vo, etc.) al neutro de una red trifásica se realizó una simulación empleando el software de simulación de Matlab-Simulink como se muestra en la Figura 8.

Se utilizó como carga no lineal 3 rectificadores monofásicos de 4 pulsos con una carga RL. En la Figura 9 se muestra los armónicos de secuencia cero en el neutro.

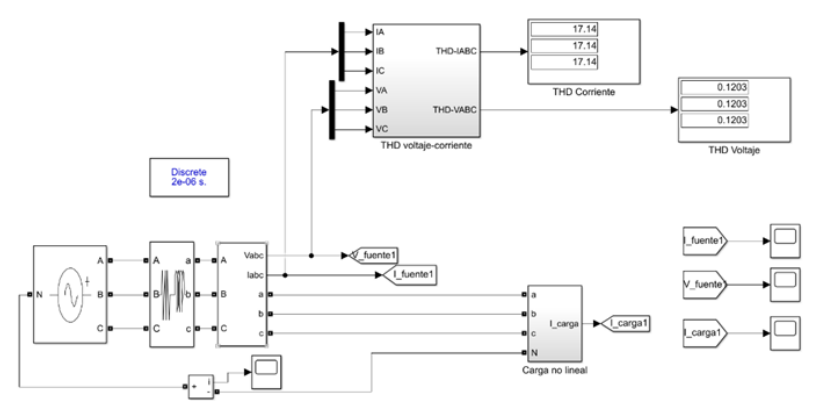

Figura 8 Sistema simulado por simulink.

Fuente: Elaboración Propia

DOMINGUEZ-DE LA CRUZ, Joel, MARTÍNEZ-RODRÍGUEZ Guillermo Miguel, GARRIDO-MELENDEZ, Javier y JIMÉNEZRIVERA Jesús. Diseño de reingeniería del sistema de puesta a tierra de un transformador de 300 KVA. Revista de Ingeniería Tecnológica. 2019 


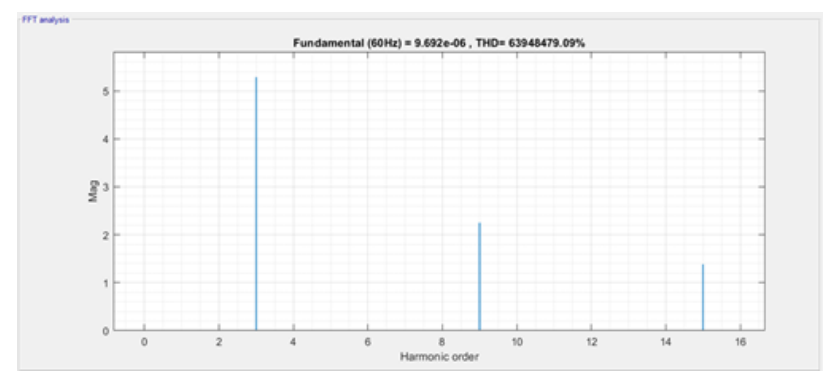

Figura 9 Sistema simulado por simulink. Fuente: Elaboración Propia

\section{Análisis del SPT}

El SPT se encuentra conectado en paralelo con el neutro del transformador, al destapar el registro del transformador se observó que solo cuenta con dos varillas interconectadas entre sí con una separación aproximada de $\mathrm{dv}=30 \mathrm{~cm}$ como se muestra en la Figura 10, se considera que el problema es el SPT. Para verificar las condiciones de este, se optó por hacer mediciones de resistencia a las varillas. Se utilizó un probador de electrodos de conexión a tierra. El método de wenner se utilizó para la medición de las varillas. La resistencia de las varillas dio un valor de $\mathrm{R}=8 \Omega$ como se muestra en la Figura 13, mientras que la norma [2] dicta que el valor mínimo son $5 \Omega$.

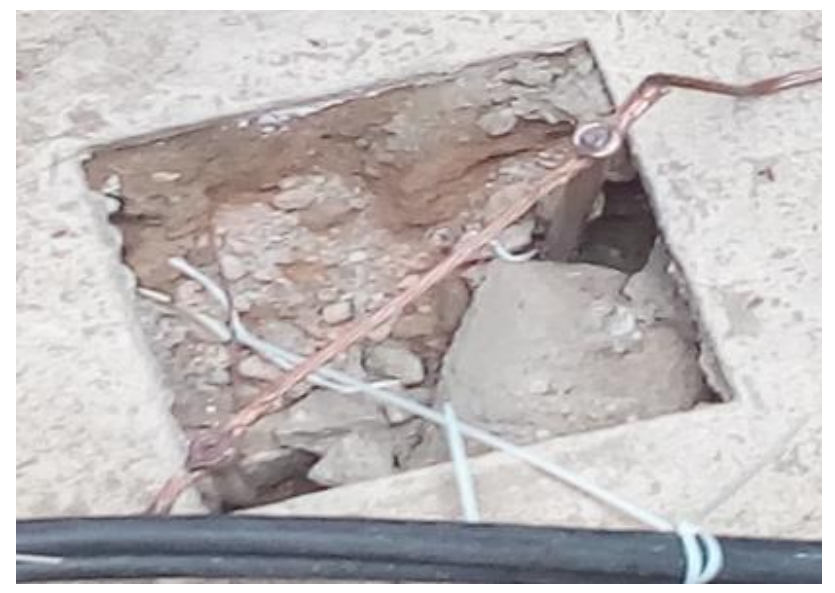

Figura 10 varillas del sistema de puesta a tierra Fuente: Elaboración Propia

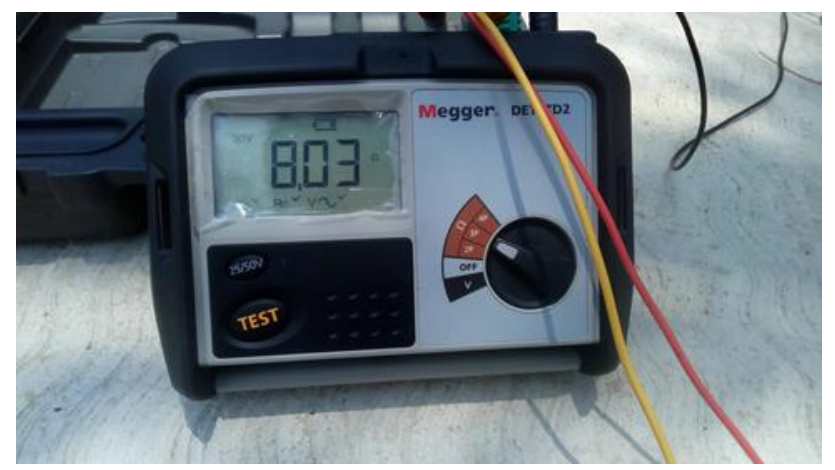

Figura 11 resultado del equipo de medición Fuente: Elaboración Propia
Por medio de la formula (1) se calcula la resistividad $\rho$ del terreno.

$\rho=(2 \pi)(d v)(R)=150.79 \Omega m$

donde: $\mathrm{dv}$ es la distancia entre varillas y $\mathrm{R}$ es la resistencia medida.

Debido a que el transformador tiene una resistencia muy alta en el sistema de tierra además de estar ubicado en una zona donde transitan estudiantes se consideró necesario calcular y simular los voltajes de contacto y de paso, con ayuda del software ETAP.

El voltaje de paso se genera entre los pies de una persona mientras se está inyectando corriente en el área en que se desplaza, dados los gradientes de potencial en la superficie del suelo. El voltaje de contacto es el que existe entre la mano y ambos pies de una persona, es decir, la diferencia entre el potencial de tierra y el potencial de la superficie en el punto donde la persona que está de pie toca con sus manos una estructura puesta a tierra [6].

Los datos para considerar son: El cortocircuito que se calcula con la formula (2), el peso promedio de $70 \mathrm{Kg}$, el tiempo de falla de 0.08 s. y el X/R de $2.5 \%$. Obteniendo el resultado mediante el software ETAP de la Figura 14.

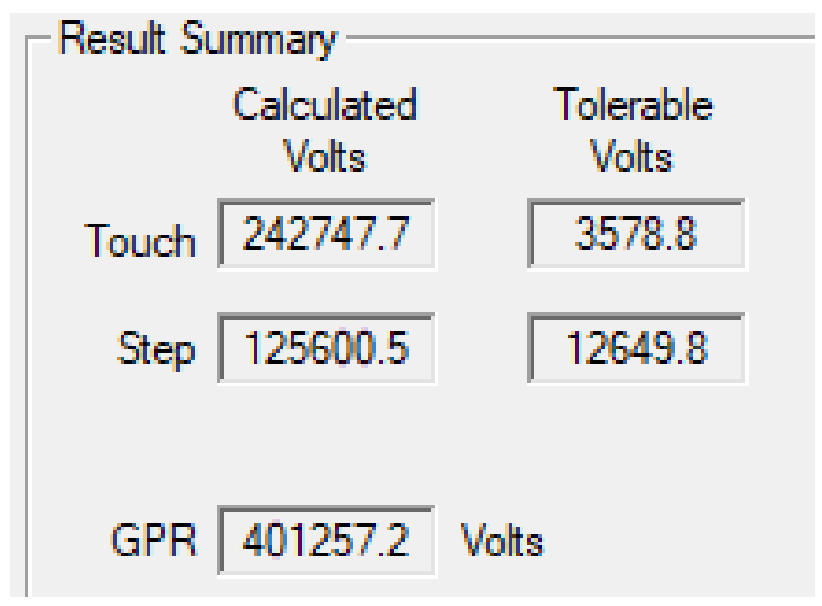

Figura 12 Resultados de los cálculos del estado actual ETAP

Fuente: Elaboración Propia

El potencial de contacto de $242747.7 \mathrm{~V}$ y el potencial de paso de $125600.5 \mathrm{~V}$ exceden el valor tolerable para el ser humano como se muestra en la Figura 12. 
En las gráficas de las simulaciones se muestra la distancia en metros en el eje $\mathrm{x}$ y el voltaje en el eje y. En la figura 13 se muestra la simulación del potencial de contacto, se obtuvo niveles que exceden el valor tolerable para el ser humano. En los óvalos se encuentran las varillas del SPT, se observa que en las varillas es la zona con menos voltaje y mientras que se aleja esta aumenta considerablemente. En la simulación no existe zona segura, los colores solo muestran los diferentes niveles de voltaje.

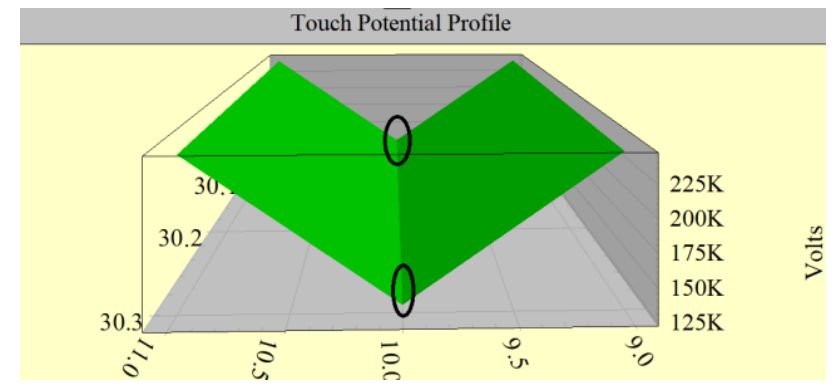

Figura 13 Potencial de contacto en el estado actual ETAP Fuente: Elaboración Propia

En la Figura 14 se muestra la simulación del potencial de paso realizado mediante el software ETAP, las varillas son el punto más alto de voltaje, se muestran como óvalos, el cual disminuye mientras se aleja, sin embargo, no hay zonas seguras.

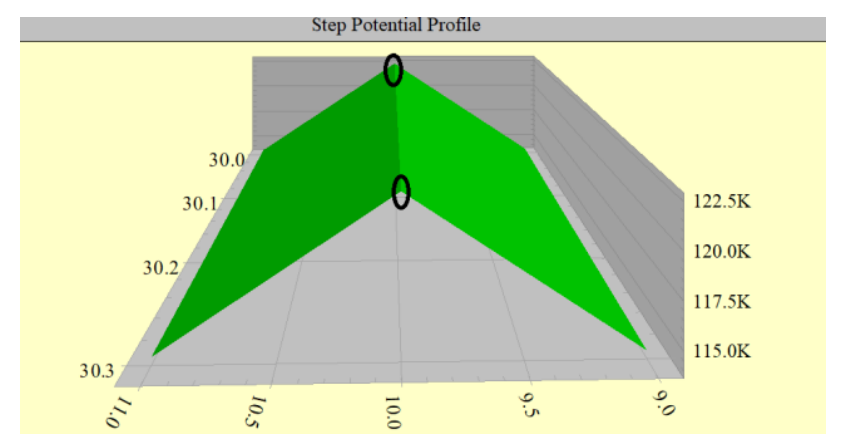

Figura 14 Potencial de paso en el estado actual ETAP Fuente: Elaboración Propia

Con los cálculos y simulaciones obtenidos se llegó a la conclusión que los potenciales de contacto y de paso no son los adecuados para el ser humano.

Los armónicos medidos también afectan debido que a medida que aumenta la frecuencia, aumenta el módulo de impedancia de puesta a tierra [7], por lo que se propone una malla de puesta a tierra al trasformador.

\section{Diseño de reingeniería del SPT}

En esta etapa se diseña una malla de puesta a tierra del transformador en donde se considerarán los siguientes puntos:

\section{- Cálculos de la malla \\ - $\quad$ Potencial de contacto en ETAP \\ - $\quad$ Potencial de paso en ETAP}

La malla elaborada en el software ETAP cuenta con una dimensión de 12 x 12 m con área de $A=144 \mathrm{~m}^{2}$, teniendo 5 conductores en el lado horizontal y 5 en el vertical. Las varillas se encuentran en varias intersecciones de la malla siendo un total de 21 varillas con una longitud de 3.05 metros como se muestra en la Figura 15

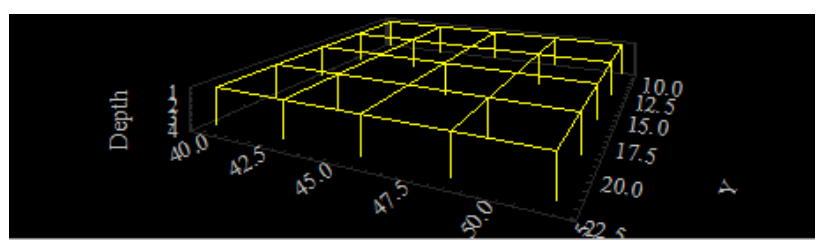

Figura 15 Malla de puesta a tierra ETAP

Fuente: Elaboración Propia

Se propone tener una capa superficial de grava de $25 \mathrm{~cm}$ con una resistividad superficial $\rho s=8534 \Omega$-m dada por ETAP.

\section{Cálculos de la malla del SPT}

Los cálculos se basaron de acuerdo con la norma IEEE 80. La corriente de cortocircuito Icc se calculó con la fórmula de bus infinito siendo I=787.296 A la corriente nominal de placa del transformador.

$I C C=\frac{I}{Z}=17115.13 \mathrm{~A}$

El primer paso para el diseño de la malla de puesta a tierra es calcular el calibre del conductor de puesta a tierra $\mathrm{A}$ con la siguiente formula:

$$
A=\frac{I_{c c}}{\sqrt{\log _{10}\left(\frac{T_{m}-T_{a}}{234+T_{a}}+1\right)}}
$$

$A=20601.76$ Circular Mils

Donde:

$I_{c c}$-Corriente de cortocircuito

$\mathrm{Tm}$-Temperatura máxima de fusión $=1084^{\circ} \mathrm{C}$

$\mathrm{Ta}$ - Temperatura ambiente $=35^{\circ} \mathrm{C}$ 
El calibre calculado es el 7 AWG $\left(10.55 \mathrm{~mm}^{2}\right)$. De acuerdo con la norma oficial mexicana, el calibre mínimo del conductor del electrodo de tierra se determina por medio de la tabla 250-66. La norma ANSI/IEEE std 80 recomienda como calibre mínimo el 4/0 AWG $(11.684 \mathrm{~mm}=0.011684 \mathrm{~m})$ por lo que se tomará este calibre.

El siguiente paso es calcular la longitud L, con esta longitud se mantendrá una tensión de malla dentro de los límites de seguridad, para esto primero se debe calcular los coeficientes $\mathrm{n}$, $\mathrm{Km}$ y $\mathrm{Ki}$

$$
\begin{aligned}
& n=N o . c p-2=3 \\
& k_{m}=\frac{1}{2 \pi} \ln \left(\frac{D^{2}}{16 * h * D_{c}}\right)+\frac{1}{\pi} \ln \left[\frac{3}{4} \times \frac{5}{6} \times \frac{7}{8}\right] \\
& K m=0.481 \\
& k_{i}=.65+.172 * \text { No.cp } \\
& k_{i}=1.166 \\
& L=\frac{k_{m} * k_{i} * \rho * I_{c c} * \sqrt{t}}{116+.117 * \rho_{s}} \\
& L=351.58 \mathrm{~m}
\end{aligned}
$$

Donde:

No. $c p$ - número de conductores en paralelo $=5$

$\mathrm{D}$ - Espaciamiento entre conductores $=3 \mathrm{~m}$

$\mathrm{h}$ - profundidad de enterramiento $=0.7 \mathrm{~m}$

Dc - Diámetro del calibre

$\rho_{s}-$ Resistividad superficial

Procedemos a sumar las distancias de las varillas y los conductores resultando una distancia total DT. En la tabla 1 se muestra la suma total.

\begin{tabular}{|l|l|l|}
\hline \multicolumn{1}{c}{ Cantidad } & \multicolumn{1}{c|}{ Longitud } & \multicolumn{1}{c|}{ Total } \\
\hline 21 varillas & 3.05 metros & $64.05 \mathrm{~m}$ \\
\hline 10 conductores & 12 metros & $120 \mathrm{~m}$ \\
\hline & & $184.05 \mathrm{~m}$ \\
\hline
\end{tabular}

Tabla 1 suma de total de longitud Fuente: Elaboración Propia

Siendo L mayor que la distancia total, se considera aceptable por no exceder los límites de seguridad.

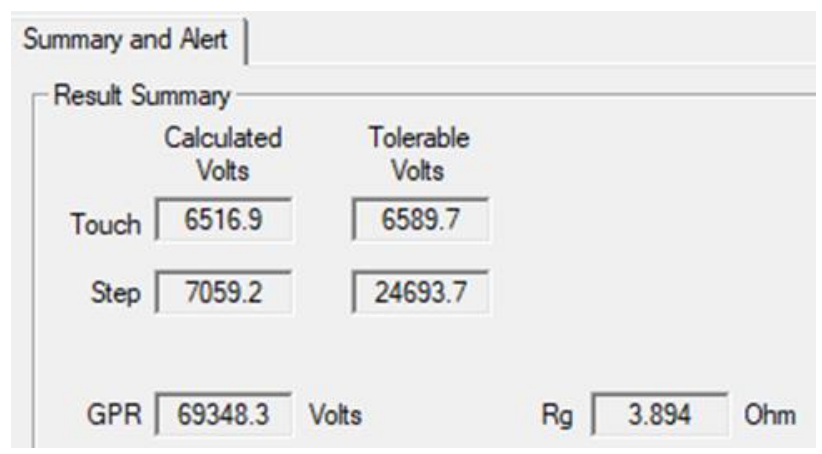

Figura 16 Cálculos obtenidos de ETAP

Fuente: Elaboración Propia

\section{Potencial de contacto}

Con el software ETAP se calcularon los potenciales de contacto y de paso del nuevo diseño como se muestra en la Figura 16.

Los valores calculados son:

- $\quad$ Potencial de contacto de $6516.9 \mathrm{~V}$ y el valor tolerable de $6589.7 \mathrm{~V}$.

- $\quad$ Potencial de paso de 7059.2 V y el valor tolerable de $24693.7 \mathrm{~V}$.

En la Figura 17 se muestra la simulación del potencial de contacto. Se observa que el punto más seguro es el centro de la malla y mientras se aleja del centro el voltaje aumenta, sin embargo, los voltajes se encuentran por debajo del valor tolerable.

\section{Potencial de paso}

El potencial de contacto simulado como se muestra en la Figura 18, muestra un voltaje bajo en el área de la malla mientras que esta se eleva en los extremos, sigue por debajo del tolerable.

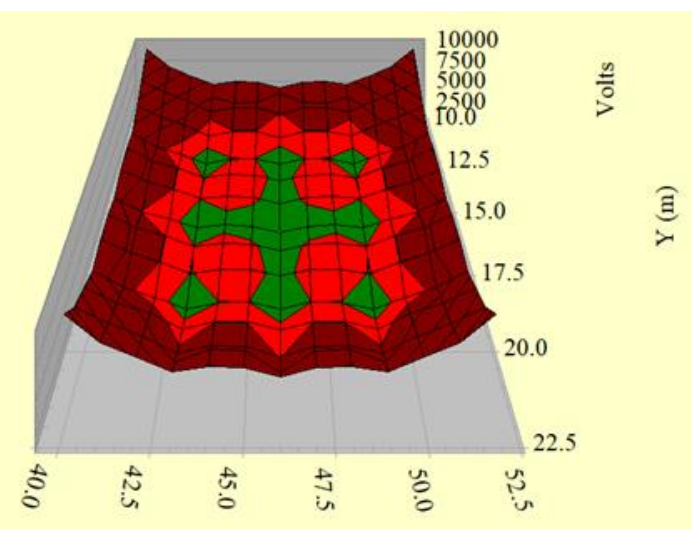

Figura 17 Simulación del potencial de contacto ETAP Fuente: Elaboración Propia 


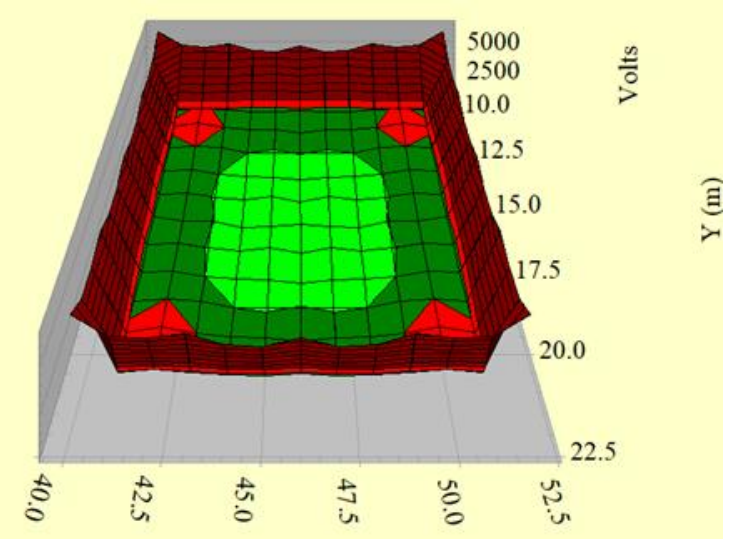

Figura 18 Simulación del potencial de paso ETAP Fuente: Elaboración Propia

\section{Resultados}

En el análisis de los armónicos se detectaron fallas en el neutro, la onda sinusoidal del neutro se ve afectada debido a los armónicos de secuencia cero. Las simulaciones con simulink comprobaron que las cargas no lineales pueden afectar al neutro. Las altas frecuencias afectan a la impedancia del SPT por lo que las mediciones realizadas a las varillas del SPT mostraron una resistividad muy alta.

Debido a que se supuso que la causa de las fallas es el SPT se optó por calcular los valores de potenciales de paso y de contacto, resultando en una diferencia de potencial peligrosa para el ser humano.

Con el diseño de reingeniería se calculó y se simuló la malla y los potenciales de paso y de contacto mostrando diferencias de potenciales tolerables para el ser humano.

\section{Agradecimientos}

Se agradece a la Facultad de Ingeniería de la Universidad Veracruzana campus Coatzacoalcos por el apoyo brindado para la realización de este trabajo.

\section{Conclusiones}

La instalación inadecuada del SPT afecta a los transformadores, lo que provoca que se tenga una resistencia alta por lo que el flujo de corriente no es óptimo, como consecuencia se obtienen fallas en el neutro, calentamiento del equipo y potenciales de paso y de contacto peligrosos para el ser humano.
En el diseño del SPT se platea mejorar el flujo de la corriente de falla por medio de una malla de puesta a tierra, esto se comprobó con simulaciones con el software ETAP. Este diseño se pretende implementar físicamente en el futuro.

\section{Referencias}

[1] IEEE Guide for Safety in AC Substation Grounding. IEEE Std 80-2000.

[2] Norma Oficial Mexicana. NOM-001-SEDE2012. Instalaciones eléctricas.

[3] Resistencia de puesta a tierra FLUKE, Principios, métodos de comprobación y aplicaciones.

[4] Qipeng Song. A Novel Approach to Eliminate Zero-sequence Harmonics Current. Key Laboratory of Power System Protection and Dynamic Monitoring and Control under Ministry of Education University. Beijing, China.

[5] Luis Fernando Gallego B. (2003). Armónicos y problemas de "power quality" en el conductor de neutro de sistemas trifásicos. Scientia et Technica.

[6] Pablo Días. (2001). Soluciones prácticas para la puesta a tierra de sistemas eléctricos de distribución. México: McGraw-Hill.

[7] Lijun Zhou. Calculation Method for Harmonic Impedance of Traction Substation Grounding Grid. Chengdu, China. 